\title{
TIME-RESOLVED MICROWAVE SPECTROSCOPY OF HIGH ELECTRON MOBILITY GaAs/AlGaAs STRUCTURES
}

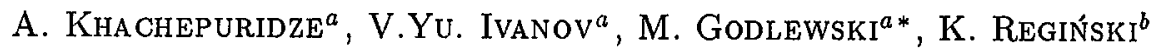 \\ AND M. BUGAJSKI ${ }^{b}$ \\ anstitute of Physics, Polish Academy of Sciences \\ Al. Lotników 32/46, 02-668 Warsaw, Poland \\ ${ }^{b}$ Institute of Electron Technology, Al. Lotników 32/46, 02-668 Warsaw, Poland
}

\begin{abstract}
Mechanism of the optical detection of cyclotron resonance via emission from 2D electron gas in modulation doped quantum wells and in high electron mobility structures of $\mathrm{GaAs} / \mathrm{AlGaAs}$ is discussed based on the results of time-resolved optical detection of cyclotron resonance. An important role of impact ionization processes is demonstrated. We also show that microwave radiation destroys emission enhancement at the Fermi level and the relevant mechanism is proposed.
\end{abstract}

PACS numbers: $73.20 . \mathrm{Dx}, 76.40 .+\mathrm{b}, 76.70 . \mathrm{Hb}$

The technique of the optically detected cyclotron resonance (ODCR) was recently used for identification of recombination transitions of $2 \mathrm{D}$ carriers in low-dimensional structures [1]. In this work we present the results of new ODCR investigations of $2 \mathrm{D}$ carriers in low-dimensional GaAs/AlGaAs structures. We discuss effects of hot carriers, heated by microwave radiation at cyclotron resonance (CR) conditions, on photoluminescence (PL) emission from either high quality modulation doped quantum well (QW) or from high electron mobility (HEM) GaAs/AlGaAs structures. Recombination transitions of $2 \mathrm{D}$ electrons, accumulated either at the GaAs/AlGaAs notch potential in HEM structures or from GaAs QW, are identified and their modifications induced by microwave radiation are studied. Effects of microwave radiation on bulk GaAs emissions are also studied for comparison.

The results discussed here were taken for two types of HEM structures (samples \#1 and \#2) and for one modulation doped QW structure (sample \#3) grown by molecular beam epitaxy. (MBE) on semi-insulating GaAs substrates. Sample \#1 consisted of $0.96 \mu \mathrm{m}$ thick GaAs buffer layer followed by $5 \mathrm{~nm}$ thick Si doped GaAs layer and by $1.68 \mu \mathrm{m}$ of undoped GaAs. On the top of the GaAs

${ }^{*}$ fax: (48-22) 8430926, e-mail: godlew@ifpan.edu.pl 
buffer $16 \mathrm{~nm}$ of undoped and $55 \mathrm{~nm}$ of Si doped $\mathrm{Al}_{0.3} \mathrm{Ga}_{0.7} \mathrm{As}$ was grown covered with $5 \mathrm{~nm}$ of Si doped $\left(2 \times 10^{18} \mathrm{~cm}^{-3}\right)$ GaAs cap layer. Sample \#2 consisted of $1.1 \mu \mathrm{m}$ thick GaAs buffer layer followed by $16 \mathrm{~nm}$ of undoped and $55 \mathrm{~nm}$ of $\mathrm{Si}$ doped $\mathrm{Al}_{0.3} \mathrm{Ga}_{0.7} \mathrm{As}$ layer and $5 \mathrm{~nm}$ of Si doped $\left(1 \times 10^{18} \mathrm{~cm}^{-3}\right)$ GaAs cap layer. Sample \#3 was GaAs/AIGaAs QW structure grown with $0.5 \mu \mathrm{m} \mathrm{GaAs} \mathrm{buffer}$ layer. The active part of the structure consisted of an undoped $10 \mathrm{~nm}$ wide GaAs $\mathrm{QW}$ surrounded by two modulation doped $\mathrm{Al}_{0.3} \mathrm{Ga}_{0.7} \mathrm{As}$ barrier layers $(10 \mathrm{~nm}$ of undoped, $20 \mathrm{~nm}$ of Si doped $\left(1 \times 10^{18} \mathrm{~cm}^{-3}\right)$ and $100 \mathrm{~nm}$ of undoped AlGaAs). The structure was capped with $10 \mathrm{~nm}$ of undoped GaAs layer. The low-temperature sheet concentrations of $2 \mathrm{D}$ carriers and carrier mobilities were $7.3 \times 10^{11} \mathrm{~cm}^{-2}$. (\#1), $7.1 \times 10^{11} \mathrm{~cm}^{-2}(\# 2)$ and $5 \times 10^{11} \mathrm{~cm}^{-2}(\# 3)$ and $1.3 \times 10^{5} \mathrm{~cm}^{2} /(\mathrm{V} \mathrm{s})$ $(\# 1), 1.04 \times 10^{5} \mathrm{~cm}^{2} /(\mathrm{V} \mathrm{s})(\# 2)$ and $7 \times 10^{4} \mathrm{~cm}^{2} /(\mathrm{V} \mathrm{s})(\# 3)$, respectively.

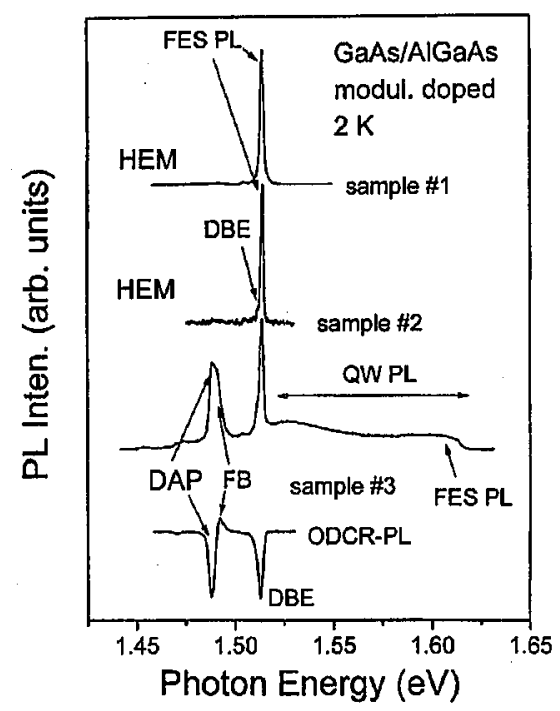

Fig. 1. Photoluminescence spectra measured at $2 \mathrm{~K}$ for two modulation doped high electron mobility GaAs/AIGaAs structures and for modulation doped single quantum well structure. The bottom curve shows PL response of the free-to-bound (enhanced) and donor-acceptor pair (quenched) emissions to the applied microwave power ( $Q$-band) at the $\mathrm{CR}$ conditions.

In Fig. 1 we show the PL spectra observed from the modulation doped QW structure (sample \#3) and from two HEM structures (\#1 and \#2). For QW structure (see Fig. 1), in addition to two "sharp" PL bands at the energy below the GaAs band gap, coming from GaAs substrate, a broad PL is observed at 1.52-1.62 $\mathrm{eV}$, which comes from GaAs QW. Due to the localization of holes in a QW, which is induced by fluctuations of a $\mathrm{QW}$ width, $k$-selection rules are relaxed and PL has a step-like shape with some enhancement at the high-energy side. This enhancement, which is a many-body effect, is called a Fermi edge singularity (FES) and is due to multiple electron hole scattering to states above the Fermi level [2]. In HEM 
structures (\#1 and \#2) step-like PL is not observed. Here a sharp PL is observed (Fig. 1) at the energies just below the GaAs band gap overlapping quasi-bulk GaAs excitonic emission of donor bound exciton (DBE). This PL is however not due to $\mathrm{DBE}$ and is related by us to recombination of $2 \mathrm{D}$ electrons accumulated at the notch potential at GaAs/AlGaAs interface. The 2D-origin of this PL is confirmed by a strong angular anisotropy of the CR signal detected via this emission. Such anisotropy (and rapid damping once magnetic field is tilted from the normal to the interface) is a signature of $\mathrm{CR}$ of $2 \mathrm{D}$ carriers [1]. The contribution of the underlying GaAs excitonic PL could be resolved from PL response to microwave power, both from the magnitude of the effect and kinetics.

In the ODCR experiment we have studied changes of the PL emission induced by an applied microwave power at the $\mathrm{CR}$ conditions. For these experiments we have used either X-band system or a newly constructed Q-band microwave system with the PL detection at the frequency of on-off modulated microwaves. We have already investigated influence of microwave radiation on PL of HEM structures $[1,3]$. In these early studies we have observed that carrier heating at the CR conditions, and for above barrier band gap excitation, can change the potential across the entire GaAs/AlGaAs heterostructure. This was explained by impact ionization of shallow donors in modulation doped AlGaAs barrier. The CR signal of electrons was then detected via a shift in a spectral position of the FES PL. In this work we study a direct influence of microwave radiation on the FES emission in samples \#1 and \#2. We analyse mechanisms of microwave-induced changes of the PL emissions. The selective response of PL emissions to heating of electrons or holes (or both electrons and holes) is observed. The response of bulk PLs in sample \#3 to microwave radiation is also studied for comparison.

Typically, modification of the $\mathrm{PL}$ emission by microwave radiation is rather weak and leads, e.g., to some enhancement of free-to-bound (FB) PL, once donoracceptor pair (DAP) PL is partly deactivated [1]. Such small modification of "bulk" PLs is also observed in the present study: DAP and DBE PLs are quenched and FB emission is enhanced at the CR conditions (see Fig. 1). The observed changes of the PL intensity are typically in the range of a few percents and synchronous detection is necessary (in phase with on-off modulated microwave power) to detect them. In contrast, we observe a large effect once detection is set at the FES PL of the HEM structures. Here the application of microwaves destroys recombination enhancement at the Fermi level and the FES PL is quenched. Quenching of the PL transitions by microwave radiation can be explained by one of the three mechanisms [1], namely by: (a) impact ionization of recombining centres, caused by interaction with hot carriers, or (b) reduced trapping rates of hot carriers, or (c) PL changes, caused by lattice heating (bolometric effect). The relative importance of these three mechanisms of the ODCR detection is still argued [1]. In our early ODCR studies we have found that the impact ionization mechanism becomes important at high frequency of microwave on-off modulation, whereas the bolometric process dominates at $\mathrm{cw}$ conditions or in experiments performed at low frequency of on-off modulation of microwaves (below $300 \mathrm{~Hz}$ in the case of $\mathrm{Si}$ [4]).

The expected differences in time of PL response for the above-mentioned mechanisms allows us to distinguish their relative importance. We have applied 


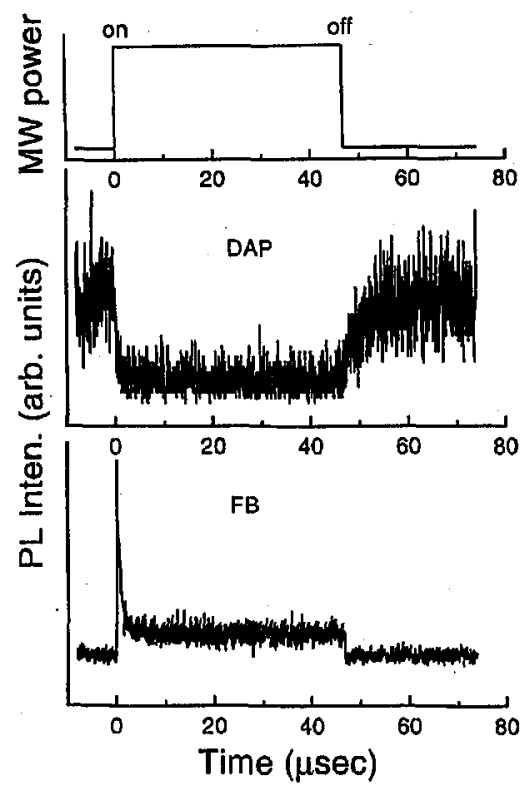

Fig. 2. Kinetics of microwave-induced changes of "bulk" free-to-bound and donoracceptor pair photoluminescence emissions in modulation doped quantum well structure of GaAs/AlGaAs.

a new technique of the time-resolved ODCR in this purpose. The kinetics of microwave-induced PL changes is studied, which allows us to analyse the dynamics of carrier heating, rate of dissipation of their excess energy and the timescale of the processes returning the system to the equilibrium. In Fig. 2 we show kinetics of microwave-induced change of the intensity of two "bulk" GaAs emissions, namely the FB and DAP PL in sample \#3. Whereas the DAP PL is quenched by turning on microwaves at electron $\mathrm{CR}$ conditions, the FB PL is first strongly enhanced and then it is slowly reduced, but to the intensity larger than that measured at microwaves off condition. Timescale of these processes is about $1 \mu \mathrm{s}$, which we explain by the time necessary for accumulation of sufficient energy by electrons (and/or holes, since CR resonances of electrons and light holes partly overlap). Hot electrons, after accumulating a sufficient energy, can impact ionise shallow donors, thus the DAP PL is quenched and the FB PL is enhanced once microwave power is turned on [1]. The following decrease in the FB PL can be related to either the reduced trapping rate of hot holes, heated by microwaves, or to the reduced recombination rate of hot electrons with holes on shallow acceptors. The latter is due to the $k$-selection rule, which prohibits radiative recombination of hot free electrons (with large $k$ values) with holes on shallow acceptors.

A much faster response to microwave radiation is observed for the FES PL. Here the PL quenching is faster than the time response of our system, i.e., it is faster than about $200 \mathrm{~ns}$. Absorption of microwave radiation by either electrons at/or close to the Fermi level or by holes immediately destroys enhancement of 
the PL oscillator strength. The CR signal detected via the FES PL is, at low microwave power, due to damped electron or/and light hole CR and, at large microwave power, changes to the heavy hole CR. The mechanism of the FES reduction by microwave power remains unknown, but the appearance of the hole-related CR signals suggests that delocalization of weakly localised holes likely explains the observed effect. A weak localization of holes was suggested as necessary for the observation of the FES PL [5]. The observed fast kinetics of the effect is in agreement with previous studies of PL kinetics, which suggest picoseconds timescale of the localization effects [6].

Concluding, the fast PL response to the microwave heating indicates a dominant role of the impact ionization processes in the ODCR. In the case of the FES $\mathrm{PL}$, the response time of the $\mathrm{PL}$ to the applied microwave radiation is much faster. We propose microwave-induced delocalization of holes as possible explanation of the effect.

This work was partly supported by the Committee for Scientific Research grants number 2 P03B 01813 and PBZ 28 11/P7.

\section{References}

[1] M. Godlewski, W.M. Chen, B. Monemar, CRC Crit. Rev. Solid State Mater. Sci. 19, 241 (1994).

[2] M.S. Skolnick, J.M. Rorison, K.J. Nash, D.J. Mowbray, P.R. Tapster, S.J. Bass, A.D. Pitt, Phys. Rev. Lett. 58, 2130 (1987).

[3] M. Godlewski, T. Lundstrom, Q.X. Zhao, W.M. Chen, P.O. Holtz, B. Monemar, T.G. Anderson, Phys. Rev. B 52, 14688 (1995).

[4] H. Weman, M. Godlewski, B. Monemar, Phys. Rev. B 38, 12525 (1988).

[5] P.O. Holtz, B. Monemar, J.L. Merz, in: Semiconductor Interfaces and Microstructures, Ed. Zhe Chuan Feng, World Scientific, Singapore 1992, p. 93.

[6] M. Bugajski, M. Godlewski, J.P. Bergman, B. Monemar, K. Regiński, M. Kaniewska, Thin Solid Films 267, 84 (1995). 